\title{
Realimentación Negativa AS: Una Aproximación a la Realimentación Negativa sin Reducción de Ganancia
}

\section{Negative Feedback AS: An Approach to Negative Feedback without Gain Reduction}

\author{
J.L. Simancas-García ${ }^{1}$ \\ 1 Profesor Investigador del grupo GIACUC adscrito al Programa de Ingeniería Electrónica de la Universidad de la Costa (CUC). \\ Email.leonardosimancas@hotmail.com
}

Recibido 01/06/13, Aceptado 30/06/2013

\section{RESUMEN}

El presente artículo es una reflexión sobre la realimentación negativa. La realimentación negativa es un clásico e importante concepto de la ingeniería electrónica que tiene un significativo número de aplicaciones, siendo una de las principales su uso en el diseño de amplificadores. La realimentación negativa ofrece múltiples ventajas, tales como el incremento del ancho de banda, reducción de la distorsión armónica, insensibilización de la ganancia del amplificador de los parámetros de los componentes activos, entre otras. Todas estas mejoras en el desempeño de los amplificadores se alcanzan al costo de ver reducida la ganancia del mismo, situación que puede ser conveniente en algunos casos, pero no en otros. Por lo anterior, en este artículo se propone una nueva forma de realimentación negativa de lazo simple con la que se tiene todas las ventajas antes mencionadas, con la novedad de no penalizar la ganancia del amplificador. Por tanto, se llevan a cabo unos desarrollos teóricos que luego son verificados con simulaciones en el software de aplicación Multisim ${ }^{\circledR}$ de National Instruments con el objeto de mostrar el funcionamiento de la aproximación propuesta en esta investigación.

Palabras clave: Amplificadores, Amplificadores Operacionales, Amplificadores de Potencia, Realimentación Negativa, Realimentación de Hawksford.

\begin{abstract}
This article is a reflexion about the negative feedback. The negative feedback is a classic and important concept for the electronic engineering and it has a significant number of applications, the main one is used in the amplifier design. Negative feedback offers many advantages, such as increasing bandwidth, reduction of the harmonic distortion, desensitization of the amplifier gain parameters of the active components, among others. The performance improvements are achieved once you can see a gain reduction, a situation that may be desirable in some cases but not in others. Therefore, in this paper it is proposed a new form of single loop negative feedback with which you have all the advantages mentioned above, with the novelty of not penalizing the gain of the amplifier. Therefore, theoretical developments were made with the purpose to verify the simulations on the Multisim ${ }^{\mathrm{TM}}$ Software of National Instruments Inc. the way it works, as you can see in this paper.
\end{abstract}

Keywords: Amplifiers, Operational Amplifiers, Power Amplifiers, Negative Feedback, Hawksford's Feedback. 


\section{INTRODUCCIÓN}

La realimentación es un importante concepto en el diseño de circuitos, donde una señal o tensión derivada de la salida es superpuesta en la entrada [1]. Esta trayectoria salida-entrada puede ser utilizada para diferentes propósitos, tales como controlar el voltaje de salida [2], control de ganancia [3], reducir la distorsión de los amplificadores [4][5][6], mejorar la estabilidad a través de la reducción del tiempo de reacción en sistemas con exceso de retardo [7]. También para crear inestabilidad [8], como en el caso de un oscilador [9][10], o mejorar la relación señal a ruido [11][12][13]. Para el caso de esta investigación resulta de interés estudiar la realimentación negativa, por ser la que permite lograr todos los propósitos antes expuestos, con excepción de la obtención de un oscilador, que requiere realimentación positiva. La realimentación negativa en amplificadores es uno de los usos más comunes que se le da a la realimentación, especialmente en los amplificadores operacionales [14]. Los propósitos básicos de utilizar realimentación negativa son controlar la ganancia y reducir la distorsión (o aumentar la linealidad) [4]. Los primeros usos de la realimentación negativa fueron reducir la distorsión en amplificadores de audio de alta potencia [15]. Una aplicación también fue en la construcción de un modulador de AM usado en transmisores de radio difusión [16][17]. Otra en los amplificadores de audio de los sistemas telefónicos [18]. La realimentación negativa es también usada para mejorar la linealidad de los amplificadores de RF, especialmente desde la introducción del transistor [19]. En la actualidad, los amplificadores de RF basados en transistores usan uno o más técnicas para obtener una linealidad superior a las provistas por la realimentación negativa [20][21]. La realimentación permite al diseñador obtener el manejo de las características del amplificador, por ejemplo la ganancia, para que no sean críticamente dependientes de los parámetros de un dispositivo activo específico que están fuera del control de quien diseña [22]. La muestra clásica de este principio es el uso de la realimentación negativa en sistemas basados en amplificadores operacionales con el objeto de que la ganancia de los circuitos sea independiente de la ganancia en lazo abierto del operacional y sea paramétricamente dependiente de componentes externos que sean controlables por el diseñador del sistema en pocas palabras, permite insensibilizar la ganancia del amplificador realimentado de los parámetros del amplificador A en lazo abierto [22].

Todas estas ventajas de la realimentación negativa se obtienen al costo de ver reducida la ganancia de los amplificadores obtenidos con ella [23]. Esta pérdida de ganancia puede ser conveniente en el caso de los amplificadores operacionales, por las razones mencionadas en el párrafo anterior [14]. Pero en la práctica existen otro tipo de amplificadores para los que la reducción de ganancia supone un costo muy alto, entonces los diseñadores deben empezar a equilibrar entre las ventajas que ofrece la realimentación y la pérdida de ganancia. Surge entonces la pregunta, ¿Es posible obtener un tipo de realimentación negativa de lazo simple que ofrezca todas las ventajas del concepto clásico sin penalizar la ganancia? Se hace patente entonces la necesidad de proponer una modificación al esquema de realimentación negativa clásico. En este artículo se propone alcanzar esta meta a través de la propuesta de una nueva forma de realimentación negativa denominada RNAS.

El trabajo está organizado de la siguiente manera. En la sección II se realiza una descripción básica sobre el concepto clásico de realimentación negativa. En la sección III se presenta una revisión de los antecedentes a este trabajo donde se habla de la realimentación de Hawksford. En la sección IV se presentan una serie de análisis teóricos sobre RNAS propuesta en este artículo. En la sección V se desarrolla un modelo de amplificador no lineal para las simulaciones que permitirán verificar los conceptos expuestos en la sección IV, y se hace una caracterización del mismo. En la sección VI se llevan a cabo una serie de simulaciones sobre el comportamiento de la RNAS frente a la realimentación negativa clásica. En la sección VII se presentan las conclusiones de este trabajo y las propuestas futuras.

\section{METODOLOGÍA}

Debido a que este trabajo de investigación es una reflexión sobre la realimentación negativa, el primer paso consistió en una revisión del tema en la literatura clásica, así como establecer su nivel de relevancia en la actualidad mediante sus aplicaciones recientes. Posteriormente se revisan los intentos previos por mejorar la realimentación negativa, y al observar que no hubo éxito en esta tarea se procede a proponer un esquema de realimentación que no adolece de las desventajas del concepto clásico, pero que conserva las ventajas de este último. Para poder constatar la validez de la aproximación propuesta en este artículo, se llevan a cabo una serie de análisis teóricos, que luego son verificados mediante los resultados de simulación en el software de aplicación Multisim ${ }^{\circledR}$ de National Instruments.

\section{REALIMENTACIÓN NEGATIVA CONVENCIONAL}

Para un concepto clásico como el de la realimentación, referencias también clásicas resulta una excelente fuente de información, por ende para presentar las ideas iniciales, se utilizará la notación clásica de los primeros trabajos realizados por los pioneros en este tema, tales como Black, Blackmann, Terman, Bode, entre otros [24-26][27][28]. Conceptualmente, los amplificadores realimentados son muy simples. En la figura 1 se puede observar este hecho. Una porción de la salida es muestreada y reintroducida a la entrada. La señal de realimentación debe estar inverti- 
da en fase, de allí el nombre de realimentación negativa. Esta inversión de fase necesaria en este tipo de realimentación se realiza automáticamente, ya que la mayoría de los amplificadores de una sola etapa invierten la fase de la señal. Por tanto, todo lo que es requerido para controlar la realimentación es un divisor de tensión para proporcionar la cantidad de realimentación necesaria a la entrada. En ausencia de realimentación, la salida es simplemente la tensión de entrada multiplicada por la ganancia del amplificador.

$$
E=e A
$$

Figura 1. Diagrama de bloques de un amplificador con realimentación negativa.

Figure 1. Block diagram of a negative feedback amplifier.

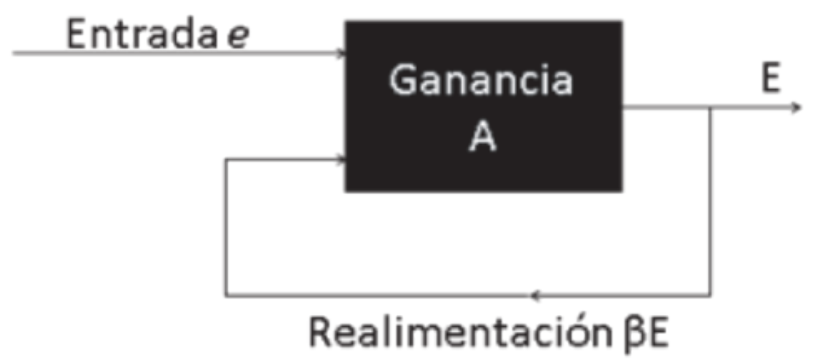

Fuente: Adaptado de [31].

Con realimentación en una proporción de $\beta E$, la señal de entrada real es $(e+\beta E)$, tal que la salida se convierte en $(\mathrm{e}+\beta \mathrm{E}) \mathrm{A}$. Debido a que la ganancia es definida como la división entre la salida y la entrada, la ganancia del sistema con realimentación es la división entre la salida y entrada reales presentada anteriormente, lo cual se reduce a [29]

\section{Ganancia con realimentación $=A /(1-A \beta)$}

Si se tiene un circuito que tiene una ganancia de tensión reducida en $20 \mathrm{~dB}$ debido a la aplicación de realimentación, entonces la ganancia es $1 / 10$, dando un factor de realimentación, $A \beta$, un valor de -9 . Con un factor de realimentación de alto valor, se puede hacer que la ganancia del amplificador con realimentación solo dependa da la red de realimentación $\beta$, permitiendo de esta manera que el diseñador controle la ganancia.

En un amplificador realimentado la distorsión es reducida debido a que la porción de la salida distorsionada que es realimentada a la entrada es re-amplificada y, ya que se encuentra fuera de fase, cancela una gran parte de la distorsión en la salida. La cantidad de reducción de la distorsión es la misma que la reducción en ganancia presentada anteriormente, $\mathrm{o}$

$$
D=d /(1-A \beta)
$$

Donde $\mathrm{D}$ es el nivel de distorsión en la salida y d es el nivel de distorsión que podría presentarse si la realimentación no estuviera presente.

En el importante libro de Sedra se menciona como el uso de la realimentación negativa mejora el desempeño de los amplificadores frente al ruido, mostrando una reducción notable en la cifra de ruido del sistema cuando es comparada con la versión sin realimentación [22]. Para una explicación básica del concepto de cifra de ruido se puede consultar el libro de Blake [9]. Rashid su libro explica que el uso de la realimentación negativa permite aumentar el ancho de banda de un amplificador, lo cual constituye un efecto positivo y conveniente en algunas aplicaciones donde se requiera [30].

Como se puede observar, la realimentación puede resultar en una gran reducción de la distorsión, una mejora del desempeño frente al ruido y un aumento del ancho de banda, en múltiples ocasiones conveniente, pero se debe recordar que todo esto se puede conseguir pagando el precio de una ganancia también es reducida. Por tanto, el amplificador debe tener un exceso de ganancia suficiente que permita la cantidad deseada de realimentación, requiriendo necesariamente que la ganancia del amplificador tienda a infinito, o en su defecto tenga una ganancia de tensión muy alta. La mayoría de los diseños de circuitos con realimentación prácticos requieren intercambios entre la ganancia y la cantidad de realimentación que puede ser aplicada para obtener la mejora requerida en las características ya mencionadas.

\section{ANTECEDENTES}

La realimentación negativa es un concepto que data del año 1927, propuesto por Harold Black y publicado en 1934 en Bell Laboratories Record [26]. Una de las dificultades principales encontradas en la realización de esta investigación es la revisión de los antecedentes, ya que estos prácticamente no existen. Sin embargo esto no debe ser mal interpretado, ya que no se intenta decir que la realimentación negativa es concepto en desuso y las referencias presentadas en la introducción de este artículo así lo demuestran, con las múltiples aplicaciones aun hoy día tiene, tales como reducción de distorsión, control de ganancia, mejora del desempeño frente al ruido, entre otras, y son trabajos en este sentido los que 
se encuentran en la literatura moderna sobre el tema, y algunas más relacionadas con nuevas metodologías utilizadas para estudiar y analizar la realimentación [31] [11] o el descubrimiento de nuevos efectos de su uso en amplificadores, tales como la medición de interferencias fuera de la banda de interés [32][33]. Lo sorpresivo es que la realimentación negativa de lazo simple no ha experimentado cambios en su estructura desde su propuesta original, sigue siendo la misma desarrollada por Black y es la utilizada en las aplicaciones antes mencionadas. No obstante el concepto fue desarrollado para el diseño de amplificadores con tubos al vacio y hoy día se utilice en amplificadores con transistores, la esencia permanece intacta. Tampoco se han reportado modificaciones o mejoras a la misma, con excepción de unos artículos presentados por M. J. Hawksford [34-36], los cuales se comentaran enseguida, y la realimentación de lazos múltiples. Como en este trabajo el objetivo perseguido es un esquema de realimentación de lazo simple, no se hará mención a la de lazos múltiples, aunque en la investigación que dio origen a este artículo si se estudiaron con cierto detalle según la información presentada en [37-39].

\subsection{Realimentación de hawksford}

Las investigaciones realizadas por Hawksford en el tema de los amplificadores lo llevo a elaborar reflexiones sobre la realimentación negativa. Sobre la realimentación negativa convencional se plantea una cuestión ¿Qué mecanismo se puede proponer para eliminar el requerimiento de ganancia infinita de lazo abierto para que en una aproximación asintótica la ganancia dependa solo de la red de realimentación? La mejor manera para evitar la necesidad de tan altas ganancias es no utilizar una realimentación degenerativa, sino una regenerativa, pero se debe descartar la realimentación positiva para evitar que el sistema se vuelva inestable. Una solución es utilizar realimentación negativa pero ejerciendo un control sobre la cantidad de realimentación que se añade a la señal de entrada para no llegar a un estado de degeneración total. Una aplicación práctica de esto fue llevada a cabo por Hawksford en el contexto de los amplificadores de potencia, utilizando una aproximación como la que se muestra en la figura 2. La función de ganancia con realimentación para este sistema es:

$$
A_{f}=\frac{V_{o}}{V_{i}}=\frac{A}{1+\beta(A-1)}
$$

Figura 2. Diagrama de bloques de un amplificador con la realimentación de Hawksford.

Figure 2. Block diagram of a amplifier using Hawksford's feedback.

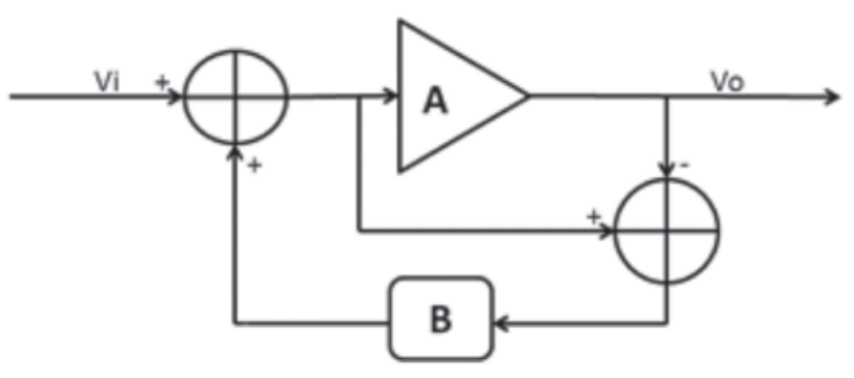

Fuente: Adaptado de [7].

Sin necesidad de recurrir a aproximaciones asintóticas, y haciendo simplemente $\beta$ igual a 1 , se puede obtener una ganancia con realimentación de $\mathrm{Af}=1$, la necesaria para el caso de amplificadores de potencia, sin importar qué valor de ganancia de lazo abierto A tenga el amplificador, porque en el momento en que la red $\beta$ se hace 1 la ganancia total con realimentación se vuelve independiente de A. De esta manera se elimina el requisito previo de que A sea infinita para poder lograr una aproximación adecuada a las necesidades de diseño. Hasta aquí se ha solucionado un gran problema para el caso particular de los amplificadores de potencia, estableciendo $\beta$ como una red de ganancia unitaria. Pero aún quedan muchas preguntas sin resolver, como por ejemplo, desde el punto de vista general (para cualquier $\beta$ y para aplicaciones diferentes a los amplificadores de potencia) ¿Qué ventajas ofrece el utilizar este tipo de realimentación modificada? ¿Cuáles son sus características principales? ¿Es realmente diferente a la realimentación negativa convencional? ¿Es simplemente una realimentación positiva convencional? Hawksford deja sin responder todas estas cuestiones en su artículo, y se dedica a hacer un análisis muy simplificado de la aproximación propuesta, y concentra toda la atención en la implementación de un circuito amplificador que se basa en este principio. El autor de este artículo sometió el esquema de realimentación propuesto por Hawksford a los siguientes análisis: de distorsión, de respuesta en frecuencia, de desempeño frente al ruido y de sensibilidad a la ganancia de lazo abierto. La conclusión de los resultados obtenidos es que la realimentación de Hawksford se comporta exactamente igual a un esquema clásico de realimentación. Estos análisis y sus conclusiones se publicarán en un trabajo posteriormente. Con las mismas ventajas y desventajas, siendo la principal entre ellas la reducción de la ganancia, sin presentarse ningún avance o mejora, salvo en su aplica- 
ción particular a los amplificadores de potencia ya mencionada. Por lo anterior, se hace patente proponer una modificación a la realimentación negativa clásica que no afecte la ganancia general del amplificador, y que resulte útil en el diseño de circuitos con amplificadores en los que se requieran todas las ventajas que ofrece la realimentación negativa convencional.

\section{REALIMENTACIÓN NEGATIVA AS}

A diferencia de la realimentación negativa ordinaria, donde una porción de la ganancia disponible de un amplificador es gastada en la obtención de los beneficios de la realimentación negativa, la propuesta presenta en este trabajo es un tipo de realimentación con el cual no ocurren tales reducciones en la ganancia directa. Se ha decidió llamar a esta nueva aproximación Realimentación Negativa AS $^{1}$ (RNAS). Para llegar a este esquema de realimentación se siguió la misma lógica seguida por Hawksford de no realimentar de manera degenerativa sino regenerativa, y para ello se amplifica el error y se suma a la entrada. Con RNAS, una porción de la señal de salida de un amplificador, que bien puede ser de una sola etapa, es sustraída de la señal de entrada, entonces la diferencia resultante es amplificada en un circuito externo cuya ganancia debe ser igual a la pérdida de ganancia que se sufriría en caso de utilizar un esquema de realimentación negativa convencional. Este error amplificado es luego sumado a la señal de entrada. De esta manera en lugar ir reduciendo la entrada efectiva que llega al amplificador, fenómeno que se manifiesta con una reducción de la ganancia de lazo cerrado (fenómeno conocido como degeneración), la entrada efectiva del amplificador en lazo abierto se mantiene constante lo que evita penalizar la ganancia. En esta sección se va a discutir sus ventajas y desventajas con algún detalle.

Figura 3. Realimentación negativa AS.

Figure 3. Negative Feedback AS.

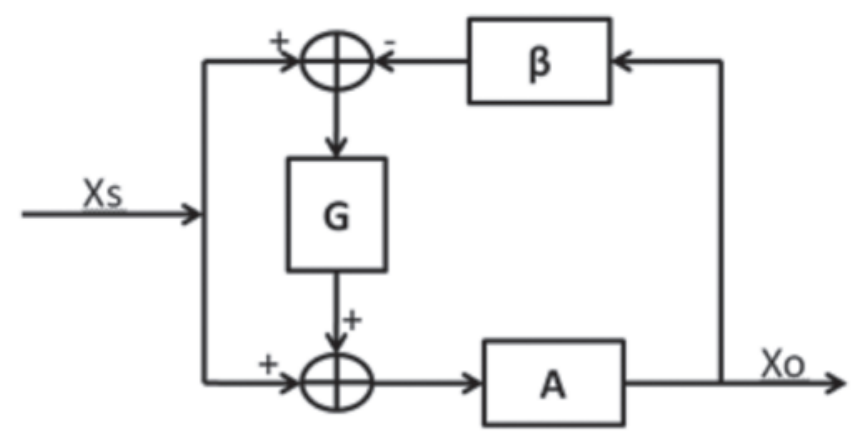

Fuente: Elaboración propia.
La figura 3 muestra un diagrama de bloques de un circuito general que utiliza RNAS. Primero se debe considerar que el circuito, la ganancia para la cual ha sido estabilizado, la distorsión y la impedancia de salida que ha sido reducida, y la respuesta en frecuencia la cual ha sido mejorada, tiene una ganancia numérica de frecuencia baja de $A_{0}$. Se debe pensar inicialmente en este esquema como función de ganancias numéricas positivas $A$ y $G$ de frecuencia baja, en lugar de ganancias fasores complejas en función de la frecuencia $A(f)$ y $G(f)$.

Utilizando el álgebra de bloques para analizar este circuito se tiene que la ganancia de lazo cerrado es

$$
\frac{X_{0}}{X_{S}}=A_{F}=\frac{A(1+G)}{1+\beta A G}
$$

Lo siguiente que se debe considerar es que la red $\beta=\mathrm{A}^{-1} \mathrm{y}$ la ganancia de lazo cerrado queda entonces

$$
A_{F}=\frac{A(1+G)}{(1+G)}=A
$$

Con lo que se puede concluir que la ganancia de lazo cerrado sigue siendo igual en valor a la ganancia de lazo abierto. La forma de utilizar esta aproximación es como se indica a continuación: se diseña un amplificador realimentado negativamente de la forma convencional, encontrando la red de realimentación $\beta$ que permita obtener las especificaciones buscadas para una ganancia de lazo abierto $A$. Esto se hace con el fin de obtener la pérdida de ganancia de lazo cerrado debido al uso de la realimentación negativa, y determinar la ganancia que debe tener el amplificador $G$. Como se dijo antes, se hace que la red $\beta$ sea igual al inverso de la ganancia de lazo abierto $A$ y de esta manera conseguimos un amplificador con RNAS que no ha sufrido reducción de ganancia de lazo cerrado. Una vez establecida esta aproximación propuesta, se debe realizar un análisis de sensibilidad a la ganancia de lazo abierto $A$, un análisis de relación señal a ruido, un análisis sobre la respuesta en frecuencia del amplificador, y finalmente un análisis cualitativo de distorsión.

\subsection{Sensibilidad a la ganancia de lazo abierto}

Se parte de la ecuación de ganancia de lazo cerrado (1) se llega a que la sensibilidad de la ganancia de lazo cerrado con respecto a la ganancia de lazo abierto es

Esta variante de la realimentación negativa recibe el nombre AS en honor a la hija del autor de este artículo, Abigail Simancas. 


$$
\frac{\delta A_{F}}{A}=\frac{\frac{\delta A}{A}}{1+G}
$$

Con lo que se puede concluir que la sensibilidad del amplificador realimentado con RNAS tiene la misma sensibilidad del amplificador realimentado convencional. Esto es así porque $G$ debe tener una ganancia que sea igual a la reducción de la ganancia en el amplificador por el uso de realimentación convencional, y el denominador de la ecuación (3) será por tanto igual al factor de realimentación.

\subsection{Relación señal a ruido}

$\mathrm{Al}$ igual que con la realimentación negativa ordinaria, el proceso de mejora de la relación señal a ruido solo será posible bajo ciertas condiciones. La principal de estas condiciones es la posibilidad de anteceder el amplificador bajo análisis de otro amplificador limpio (libre de ruido) para poder establecer el lazo de RNAS, como se muestra en la figura 4. Para el caso en cuestión, $A 2$ es el amplificador libre de ruido y $A 1$ es el amplificador afectado por el ruido, y al que se le quiere aumentar la relación señal a ruido.

Figura 4. Esquema de la RNAS para encontrar la SNR. Figure 4. RNAS scheme to find the SNR.

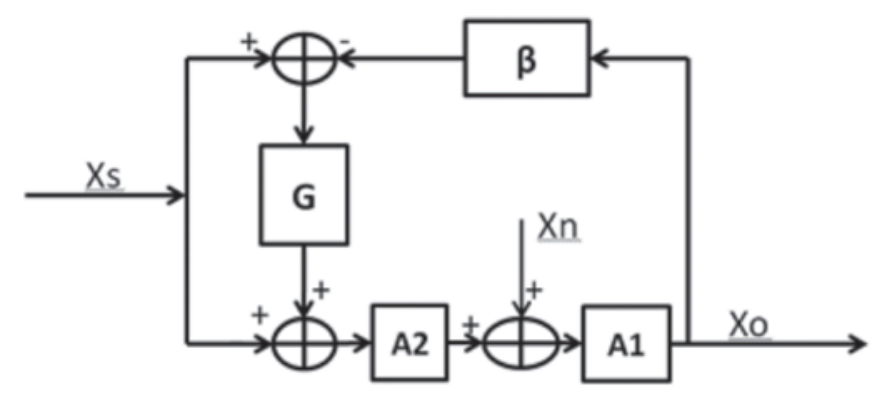

Fuente: Elaboración propia.

Utilizando el álgebra de bloques para analizar el flujo de señales en el circuito de la figura 4 se obtiene que la relación señal a ruido en la salida del amplificador con respecto a la que se tiene en la entrada es la siguiente

$$
\left(\frac{S}{N}\right)_{0}=\frac{\frac{(1+G) A_{2}}{\left(1+\beta A_{2} G\right)} X_{S}}{\frac{A_{1}}{\left(1+\beta A_{2} G\right)} X_{n}}=\frac{(1+G) A_{2}}{A_{1}} \frac{X_{S}}{X_{n}}=\frac{(1+G) A_{2}}{A_{1}}\left(\frac{S}{N}\right)_{i}
$$

Como se puede observar de esta última expresión, el resultado no es muy claro, pero cabría esperar una mejora en la relación señal a ruido con el uso de este amplificador con RNAS siempre y cuando la ganancia del amplificador libre de ruido sea elevada. Sin embargo esto no es importante, recuérdese que la mejora en la relación señal a ruido solo se consigue si se cumple la condición de tener un amplificador libre de ruido antecediendo al amplificador de interés, y esto es poco probable que suceda, ya que no tiene sentido gastar tiempo de diseño en obtener un amplificador libre de ruido para mejorar la relación señal a ruido de otro, en ese caso se invierte ese esfuerzo adicional en aplicar técnicas de reducción de ruido diferentes a la realimentación al amplificador de interés.

\subsection{Respuesta en frecuencia}

La realimentación negativa ordinaria incrementa el ancho de banda, como ya se dijo en otra sección de este artículo. Se debe comprobar que la realimentación negativa AS también contribuye a mejorar el ancho de banda de un amplificador. Para tal fin, se considera un amplificador simple, cuya ganancia en lazo abierto $A$ sea una función de la frecuencia, lo que puede expresarse en el dominio de Laplace como

$$
A(s)=\frac{A_{0}}{1+{ }^{s} / 2 \pi f_{H}}
$$

Donde $A_{0}$ es la ganancia a baja frecuencia de lazo abierto y $f_{H}$ es la frecuencia de corte de $3 \mathrm{~dB}$ de lazo abierto, en Hz. Por otra parte, la ganancia del amplificador $G$ también depende de la frecuencia, pero este amplificador debe tener un ancho de banda mucho mayor que el de $A$ y por tanto su ganancia se puede considerar constante para el rango de frecuencias de interés de $A(s)$. Este criterio es válido debido a la relación inversa que existe entre la ganancia y el ancho de banda, y dado que $G$ tiene una ganancia mucho menor que $A$ es posible alcanzar en él un mayor ancho de banda durante su diseño. La ganancia del amplificador realimentado en el dominio de la frecuencia es

$$
A_{F}(s)=\frac{A_{0}}{1+\frac{s}{2 \pi f_{H}\left(1+G_{0}\right)}}
$$

De donde se pueden extraer las siguientes relaciones

$$
\begin{array}{r}
A_{0 F}=A_{0}(6) \\
f_{0 F}=f_{H}\left(1+G_{0}\right)
\end{array}
$$


De las cuales se puede concluir que la ganancia a frecuencia baja para el amplificador con realimentación negativa AS es igual a la del amplificador en lazo abierto. El ancho de banda ha sufrido un escalamiento que es proporcional al factor de realimentación, como en la realimentación negativa clásica. Como se puede observar, se cumple que este esquema de realimentación aquí propuesto, deja invariable la ganancia del amplificador en baja frecuencia. Finalmente se observa que para llegar al resultado obtenido se asumió que el amplificador era uno del tipo pasa bajos, sin embargo su aplicación se puede extender a amplificadores pasa banda, donde el ancho de banda de este último se expandirá en la misma proporción aquí presentada, como se podrá evidenciar más adelante en las simulaciones.

\subsection{Análisis de distorsión armónica}

Las ideas expuestas en esta sección obedecen a las conclusiones extraídas de la revisión de [40]. Debido al uso de dispositivos no lineales, tales como los transistores, la ganancia de los amplificadores también presenta un comportamiento no lineal. Cuando una señal de tipo sinusoidal es ingresada a un sistema no lineal, en su salida aparecen componentes armónicas de la señal de entrada, las cuales provocan una deformación de la señal de interés. En síntesis, la distorsión armónica se puede definir como una alteración de la forma de onda de una señal provocada por la aparición de nuevas componentes debido al comportamiento no lineal de la ganancia del amplificador. Estas nuevas componentes espectrales son múltiplos o armónicas de las frecuencias de otras componentes de frecuencia que hacen parte de la señal original. Si un sistema tiene una ganancia no lineal, la curva que representa el comportamiento entrada - salida del circuito se puede describir con la siguiente ecuación

$$
v_{\text {sal }}=A v_{\text {ent }}+B v_{\text {ent }}^{2}+C v_{\text {ent }}^{3}+\cdots(8)
$$

Los coeficientes $A, B, C$ y así, constituyen las ganancias del sistema para esas componentes armónicas específicas. Además estos coeficientes están en función de la ganancia nominal del amplificador. Por ende, entre mayor sea la ganancia nominal mayor serán los valores de los coeficientes de las armónicas y de igual forma será mayor la distorsión. Físicamente, la distorsión se da cuando, producto de la ganancia, los niveles de las señales en el amplificador alcanzan valores que sacan a los dispositivos activos (transistores por ejemplo) de su zona de trabajo lineal, provocando la aparición de las armónicas espurias. La realimentación negativa convencional es un mecanismo básico de reducción de la distorsión ya que la realimentación reduce la ganancia del amplificador y por ende el riesgo de distorsión debido a que las señales en el amplificador no alcanzarán niveles que fuercen a los transistores a trabajar fuera de su rango de trabajo lineal [40]. La RNAS no reduce la distorsión debido a que su uso no disminuye la ganancia del amplificador, la mantiene en su valor nominal, como ya se demostró antes en este artículo.

\section{MODELO DE AMPLIFICADOR NO LINEAL}

Se consultó el libro de diseño microelectrónico de Rashid [30], y del capítulo concerniente al diseño de amplificadores se extrajo un modelo de amplificador de un ejercicio teórico, el cual es consecuente con los modelos de los clásicos [41-42].

Las especificaciones del circuito son:

- $\quad$ Ancho de Banda (BW): $1 \mathrm{kHz}$ hasta $100 \mathrm{kHz}$.

- Ganancia de voltaje de rango medio $\mathrm{A}_{\mathrm{v}}$ :-200 (se trata de un amplificador inversor).

El amplificador está conectado a una resistencia de carga $R_{L}=10 \mathrm{k} \Omega$ y la fuente de señal tiene una resistencia de fuente $R_{S}=2 \mathrm{k} \Omega$, y se modela con el siguiente circuito mostrado en la figura 5 . En este modelo el amplificador tiene una resistencia de entrada $R_{i}=2 \mathrm{M} \Omega$ y un capacitor de acoplamiento de entrada $C_{1}=158.84 \mathrm{pF}$. Este capacitor es quien determina la frecuencia de corte inferior de $3 \mathrm{~dB}$ de la respuesta en frecuencia del amplificador. El capacitor en paralelo con la salida $\mathrm{C}_{2}=159.15 \mathrm{pF}$ determina la frecuencia de corte superior de $3 \mathrm{~dB}$. El proceso de amplificación como tal se ha modelado a través de una fuente de corriente dependiente de voltaje, con una transconductancia de $20 \mathrm{~mA} / \mathrm{V}$ o $20 \mathrm{mMho}$. Esta transconductancia combinada con la resistencia de carga de $10 \mathrm{k} \Omega$ permite obtener la ganancia de -200 especificada para el amplificador. Los diodos Zener que aparecen en la salida en paralelo con la carga representan la saturación del amplificador, que se supone alimentado con $\pm 15 \mathrm{~V}$ de DC, y que a su vez modelan el comportamiento no lineal del amplificador. Una vez trazado el modelo, se establece la señal de entrada como una del tipo sinusoidal, con amplitud pico de $500 \mathrm{mV}$ y una frecuencia de $50 \mathrm{kHz}$. Usando la herramienta de simulación de circuitos eléctricos de National Instruments, Multisim ${ }^{\circledR}$ y los análisis que con ella se pueden hacer para verificar el cumplimiento de las especificaciones antes presentadas. A continuación se resumen las especificaciones del amplificador simulado:

- $\quad$ Ancho de Banda (BW): $500 \mathrm{~Hz}-100 \mathrm{kHz}$.

- Ganancia de Voltaje de rango medio A : -200.

- Saturación (no considerada inicialmente): $\pm 15.5 \mathrm{~V}$.

- Distorsión Armónica Total (THD): 33.17 \%, para una entrada de $500 \mathrm{mV}$ pico.

- Magnitud de 3ra armónica: 5,72903 V. 
De esta manera se ha caracterizado el amplificador no lineal con el que se van a desarrollar las pruebas y las simulaciones de los conceptos relacionados con la RNAS explicados en la sección anterior.

Figura 5. Modelo de amplificador no lineal.

Figure 5. Nonlinear amplifier model.

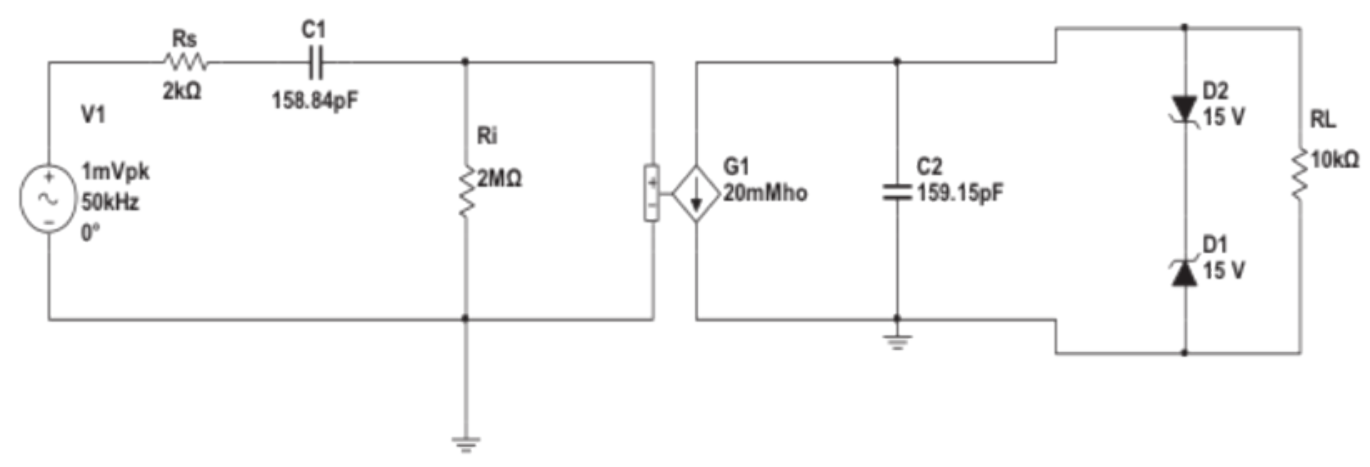

Fuente: Adaptado de [21]

\section{PRUEBAS Y RESULTADOS}

En este apartado se realizaran las pruebas que verifican que la realimentación negativa propuesta en esta investigación (RNAS) se comporta tal cual la presenta la teoría. La metodología consistirá en tomar un ejercicio de un texto de electrónica estándar sobre realimentación negativa clásica y resolverlo teóricamente, y luego simularlo para verificar que los resultados obtenidos numéricamente son válidos. Luego se resuelve el mismo ejercicio teóricamente pero aplicando la RNAS propuesta en este trabajo, y al igual que en el caso anterior, se realizara la correspondiente simulación para la comprobación de los resultados. Se llevarán a cabo 3 análisis: Sensibilidad a la ganancia de lazo abierto del amplificador, respuesta en frecuencia del amplificador, y distorsión armónica total en la salida. El análisis del desempeño frente al ruido no se lleva a cabo en este artículo debido a las irreales condiciones a las que se tendría que someter el amplificador para que una mejora pueda darse. Las simulaciones se realizan en el software de aplicación Multisim ${ }^{\circledR}$ de National Instruments.

\subsection{Realimentación negativa ordinaria}

Se utilizaran para los cálculos teóricos las especificaciones del amplificador obtenidas en la simulación presentada anteriormente. La red de realimentación negativa tendrá un valor $\beta=0.1$. El diagrama de bloques del circuito que se simulo es el que se presenta en la figura 6. Como se puede observar, ni la red de realimentación $\beta$ ni la entrada secundaria del sumador en la entrada del amplificador necesitan inversión de fase para alcanzar la realimentación negativa, ya que el amplificador $A$ es inversor, y la señal realimentada tiene fase contraria a la señal de entrada. Para este amplificador se realizan las simulaciones correspondientes a los mismos análisis realizados para el caso de lazo abierto, es decir: análisis AC para respuesta en frecuencia, análisis de sensibilidad a la ganancia de lazo abierto, y un análisis de Fourier para calcular la distorsión. Para poder realizar la comparación del amplificador realimentado negativamente con respecto al amplificador en lazo abierto, la señal de entrada conserva los mismos parámetros. El bloque de simulación A contiene al modelo de amplificador presentado en la figura 5.

Figura 6. Amplificador con realimentación negativa convencional.

Figure 6. Conventional negative feedback amplifier.

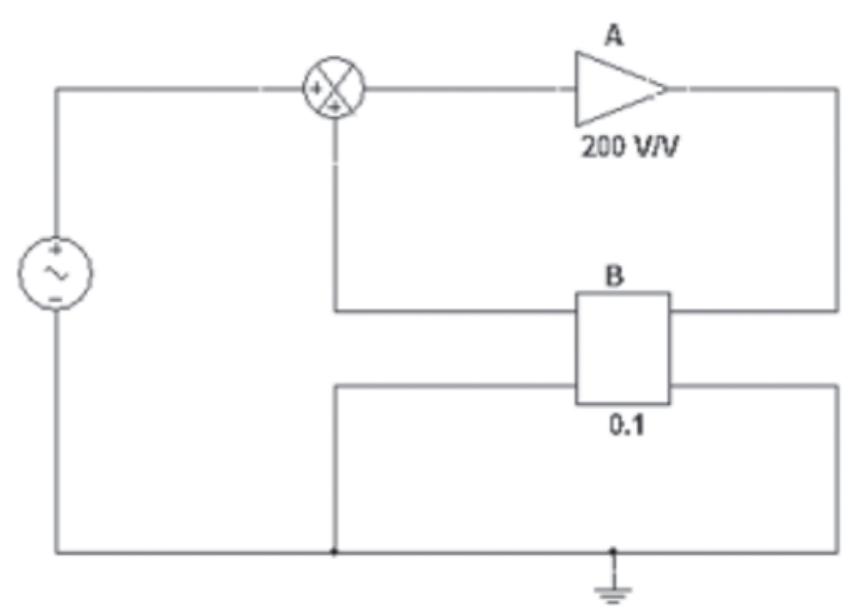

Fuente: Elaboración propia. 
El ejercicio se desarrolla para $|A|=200$ y $\beta=0.1$. El ancho de banda para el amplificador realimentado será de

$$
B W_{F}=(1+A \beta) B W=(1+200 \times 0.1)(99 \mathrm{kHz})=2079 \mathrm{kHz}
$$

Como se puede observar, el ancho de banda será llevado de 99 kHz en lazo abierto a 2079 kHz con la realimentación negativa convencional. Sin embargo, la ganancia es penalizada y tendrá un valor de

$$
A_{F}=\frac{A}{(1+A \beta)}=\frac{200}{1+200 \times 0.1}=9.52
$$

Y la distorsión armónica total tendrá un valor de

$$
T H D_{F}=\frac{T H D}{1+A \beta}=\frac{33.17 \%}{1+200 \times 0.1}=1.57 \%
$$

La sensibilidad del amplificador realimentado a la ganancia de lazo abierto con una variación del $10 \%$ es la siguiente

$$
\frac{\delta A_{F}}{A}=\frac{\frac{\delta A}{A}}{(1+\beta A)}=\frac{10 \%}{1+200 \times 0.1}=0.47 \%
$$

A continuación se resumen las especificaciones obtenidas de la simulación del amplificador realimentado y las cuales se pueden observar e la figura 7 y en la tabla 1:
- $\quad$ Ancho de Banda (BW): $23 \mathrm{~Hz}-2.1 \mathrm{MHz} . \mathrm{BW}=2077$ $\mathrm{kHz}$.

- $\quad$ Ganancia de Voltaje de rango medio A : -9.5 .

- Saturación (no considerada inicialmente): $\pm 15.5 \mathrm{~V}$.

- Distorsión Armónica Total (THD): 0.0676 \%, para una entrada de $500 \mathrm{mV}$ pico y $50 \mathrm{kHz}$.

En cuanto al análisis de sensibilidad se introdujo una variación de la ganancia de lazo abierto correspondiente al $10 \%$, es decir, se redujo de $-200 \mathrm{~V} / \mathrm{V}$ a $-180 \mathrm{~V} / \mathrm{V}$. Y la ganancia de lazo cerrado se redujo de $-9.5 \mathrm{~V} / \mathrm{V}$ a $-9.47 \mathrm{~V} / \mathrm{V}$, lo que corresponde a una variación de $0.32 \%$. Este valor de sensibilidad es aceptable y se ajusta de manera cercana al valor teórico.

Una comparación entre los resultados del simulador y los resultados teóricos da cuenta que las predicciones realizadas con las ecuaciones de la teoría son bastante cercanas en su mayoría, excepto para la THD. La razón de esta última discrepancia se encuentra en el modelo mismo. Una inspección del modelo permite concluir que este último es básicamente lineal, y que la no linealidad es introducida por los diodos Zener y esta solo se manifiesta cuando la señal de salida alcanza los límites de saturación de la característica de transferencia del amplificador. En la práctica la no linealidad de las características entrada salida del sistema se manifiestan antes de alcanzar los niveles de saturación. Eso hace que para este caso una manifestación de la distorsión dependa fuertemente del nivel de la señal de entrada al amplificador, ya que niveles de voltaje bajos que al ser aplicados el amplificador realimentado no lleven su salida a los extremos de saturación presentarán una distorsión casi despreciable. Pero, en todo caso se puede observar la reducción de la THD gracias al uso de realimentación negativa convencional.

Figura 7. Respuesta en frecuencia del amplificador utilizando realimentación negativa convencional.

Figure 7. Frequency response of the amplifier using the conventional negative feedback.

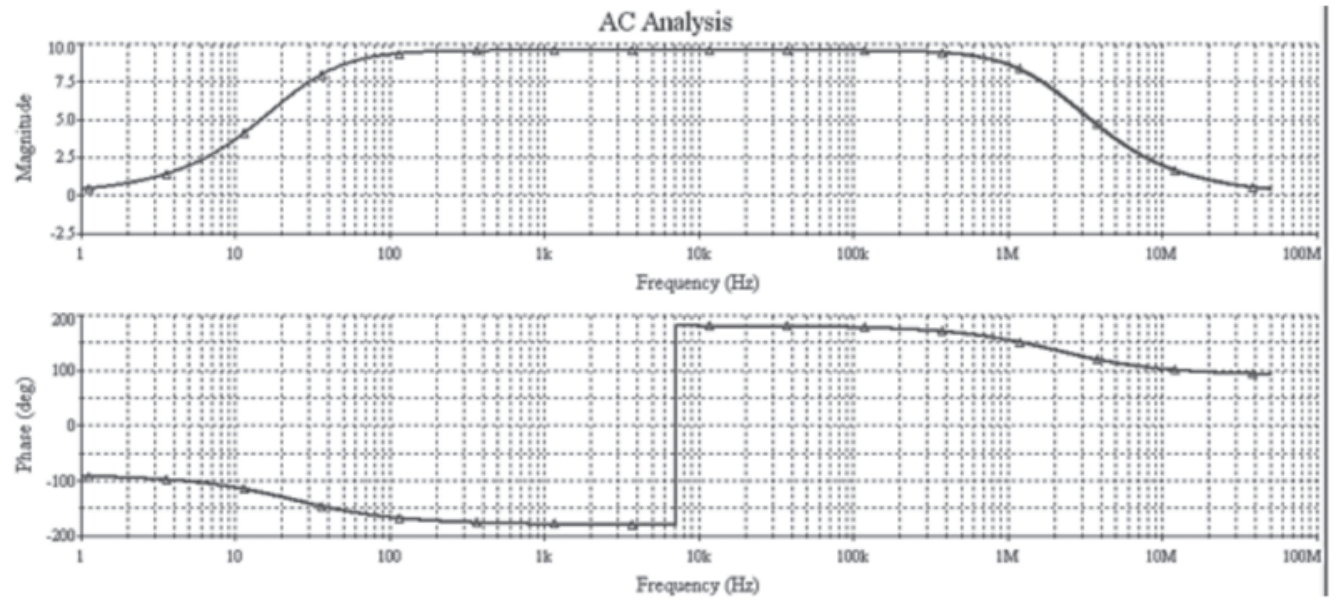

Fuente: Tomado del simulador Multisim. 
Tabla 1. Análisis de Fourier obtenido en el simulador. Table 1. Fourier Analysis obtained in the simulator.

\begin{tabular}{|l|c|}
\hline \multicolumn{1}{|c|}{ Fourier Analysis } & \\
\hline DC component: & 0.0907575 \\
\hline No. Harmonics: & 10 \\
\hline THD: & $0.0676 \%$ \\
\hline Gridsize: & 256 \\
\hline Interpolation Degree: & 1 \\
\hline
\end{tabular}

Fuente: Tomado del simulador Multisim.

\subsection{Realimentación negativa as}

En la figura 8 se presenta el esquema de bloques del circuito que se simulo para verificar el comportamiento de la realimentación negativa AS. La pérdida de ganancia del amplificador usando la realimentación negativa ordinaria es de 20 $(|\mathrm{A} / \mathrm{AF}|=|-200 / 10|)$, por tanto la ganancia del amplificador de error en el lazo de realimentación debe ser de 20. La red $\beta$ debe tener un valor de A-1, esto es, $1 / 200=0.005$, tal como se muestra en la figura 8. Para este circuito se aplicaron los mismos análisis de los casos anteriores: análisis AC para obtener la respuesta en frecuencia del amplificador realimentado, un análisis de Fourier para medir la distorsión y un análisis sensibilidad.

Los resultados teóricos esperados para el amplificador con la realimentación negativa AS son los siguientes

$$
B W_{F}=(1+G) B W=(1+20)(99 k H z)=2079 k H z
$$

Igual que en el caso de la realimentación negativa clásica. La ganancia del amplificador en el rango medio para el caso en que se use realimentación negativa modificada será de

$$
A_{F}=\frac{A(1+G)}{1+\beta A G}=\frac{200(1+20)}{1+(0.005 \times 20 \times 200)}=200
$$

En el caso de la sensibilidad a la ganancia de lazo abierto para una variación de $10 \%$ se tiene que

$$
\frac{\delta A_{F}}{A_{F}}=\frac{\frac{\delta A}{A}}{1+G}=\frac{10 \%}{1+20}=0.47 \%
$$

La sensibilidad esperada es igual a la obtenida con la realimentación negativa convencional.
Figura 8. Realimentación Negativa AS.

Figure 8. Negative Feedback AS.

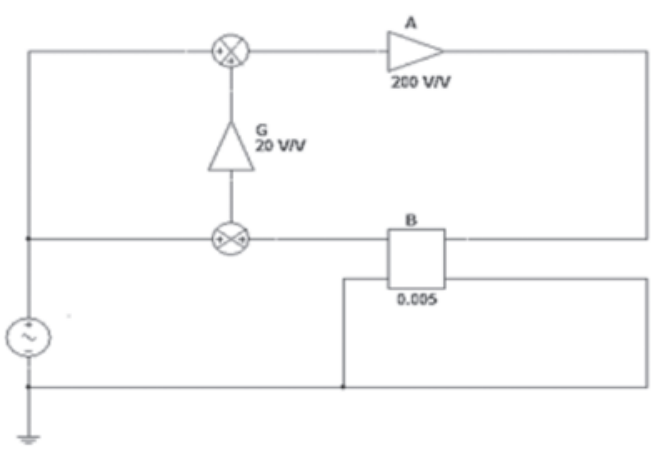

Fuente: Elaboración propia.

En el caso de la distorsión armónica total se estima que debe ser la misma. Para realizar las simulaciones se escogió como señal de entrada una del tipo sinusoidal, con una amplitud de $500 \mathrm{mV}$ pico y una frecuencia $50 \mathrm{kHz}$. Los resultados se resumen a continuación y se pueden apreciar en la figura 9 y en la tabla 2.

- $\quad$ Ancho de Banda (BW): $23 \mathrm{~Hz}-2.1 \mathrm{MHz} . \mathrm{BW}=2077 \mathrm{kHz}$.

- Ganancia de Voltaje de rango medio A : -198.

- Saturación (no considerada inicialmente): $\pm 15.7 \mathrm{~V}$.

- Distorsión Armónica Total (THD): $39.04 \%$, para una entrada de $500 \mathrm{mV}$ pico y $50 \mathrm{kHz}$.

El ancho de banda y la ganancia de voltaje de rango medio dan valores acordes con la predicción teórica. La saturación también resulto como se esperaba, debido a que esta se introdujo deliberadamente en el modelo del amplificador con el uso de los diodos Zener. La distorsión armónica obtenida fue mayor que la esperada en un $5 \%$ aproximadamente debido a que la señal de entrada en lugar de ser degenerada es regenerada en el esquema RNAS. Para verificar la sensibilidad de este esquema de realimentación a la ganancia de lazo abierto se modificó esta última de $-200 \mathrm{~V} / \mathrm{V}$ a $-180 \mathrm{~V} / \mathrm{V}$, y la ganancia de rango medio resultante fue de $-198 \mathrm{~V} / \mathrm{V}(1 \%)$. La sensibilidad obtenida fue mayor que la esperada teóricamente, pero lo suficientemente cercana para aceptar la validez de la reducción de la sensibilidad teóricamente calculada.

Tabla 2. Análisis de Fourier obtenido del simulador. Table 2. Fourier Analysis obtained in the simulator.

\begin{tabular}{|l|c|}
\hline \multicolumn{1}{|c|}{ Fourier Analysis } & \\
\hline DC component: & 0.0661707 \\
\hline No. Harmonics: & 10 \\
\hline THD: & $39.0077 \%$ \\
\hline Gridsize: & 256 \\
\hline Interpolation Degree: & 1 \\
\hline
\end{tabular}

Fuente: Tomado del simulador Multisim. 
Figura 9. Respuesta en frecuencia de la RNAS.

Figure 9. Frequency response of the RNAS.

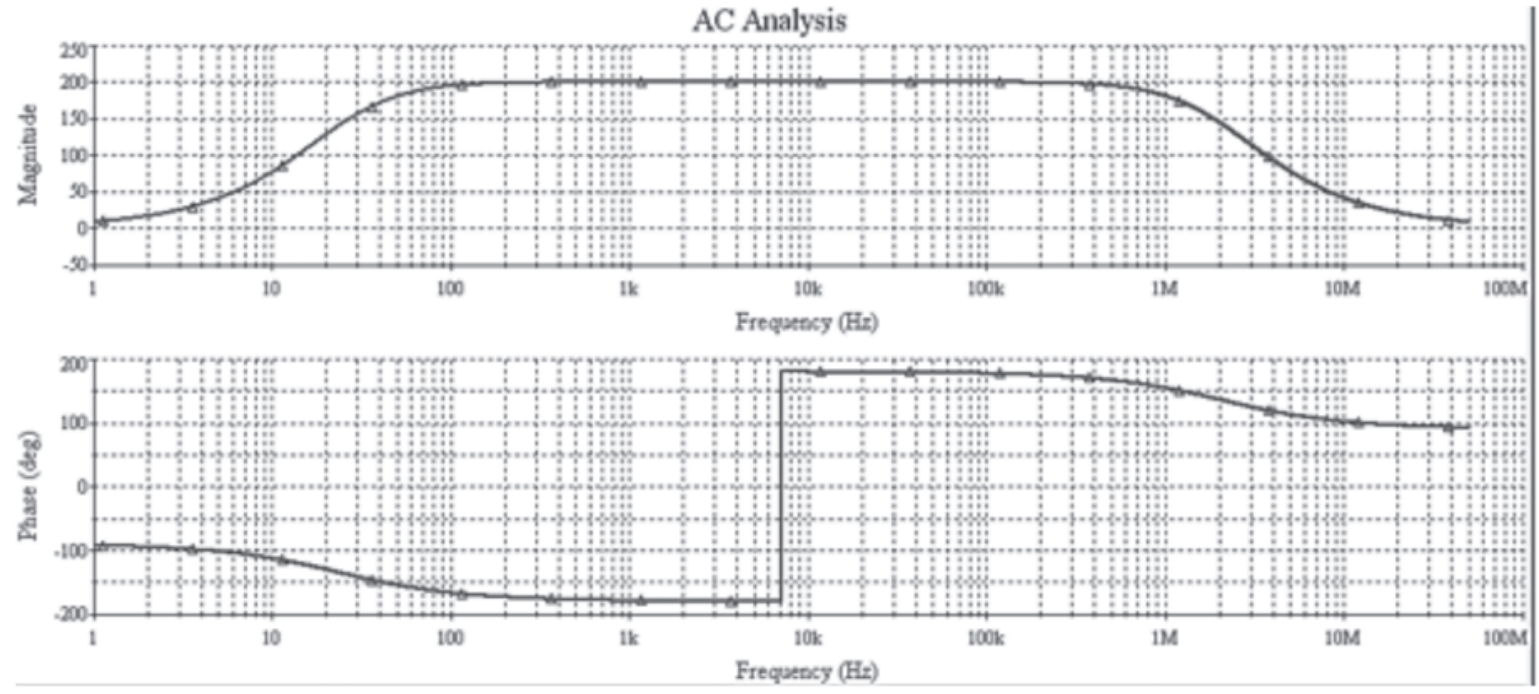

Fuente: Tomado del simulador Multisim.

\subsection{Comparación de los esquemas de realimentación}

Como el resultado más importante de esta propuesta se obtuvo en la respuesta en frecuencia, es decir, el incremento del ancho de banda del amplificador en la misma proporción que la hace la realimentación negativa con- vencional pero sin pérdida de ganancia de rango medio, a continuación en las figura 10 y 11 se presentan unas gráficas comparativas de los 2 esquemas de realimentación en este sentido. En la figura 11 se muestra como la respuesta en fase de la realimentación AS es igual a la exhibida por la realimentación negativa convencional.

Figura 10. Magnitud de la respuesta en frecuencia en dB de los 2 esquemas de realimentación.

Figure 10. Magnitude of the frequency response in $\mathrm{dB}$ of the two feedback schemes.

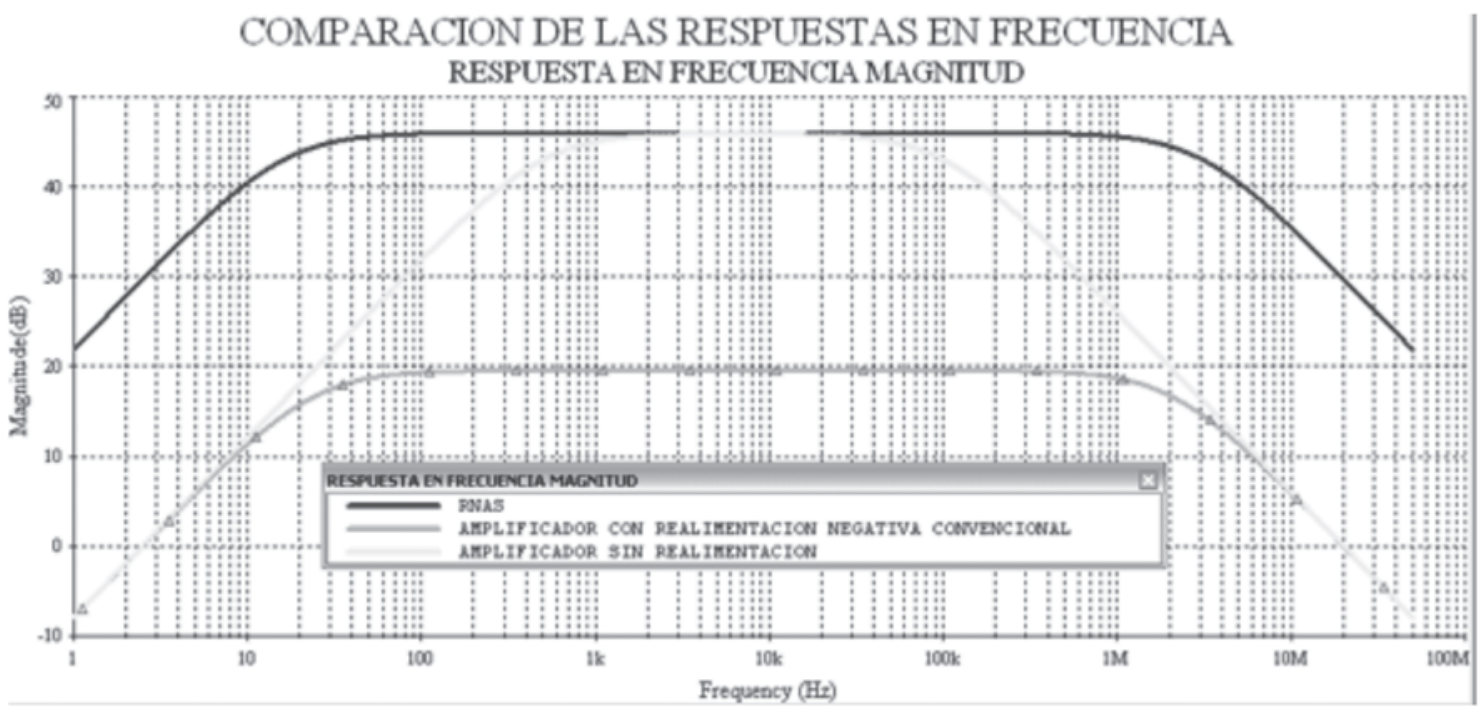

Fuente: Tomado del simulador Multisim. 
Figura 11. Fase de las respuestas en frecuencia de los 2 esquemas de realimentación estudiados.

Figure 11. Phase frequency responses of the two feedback schemes studied.

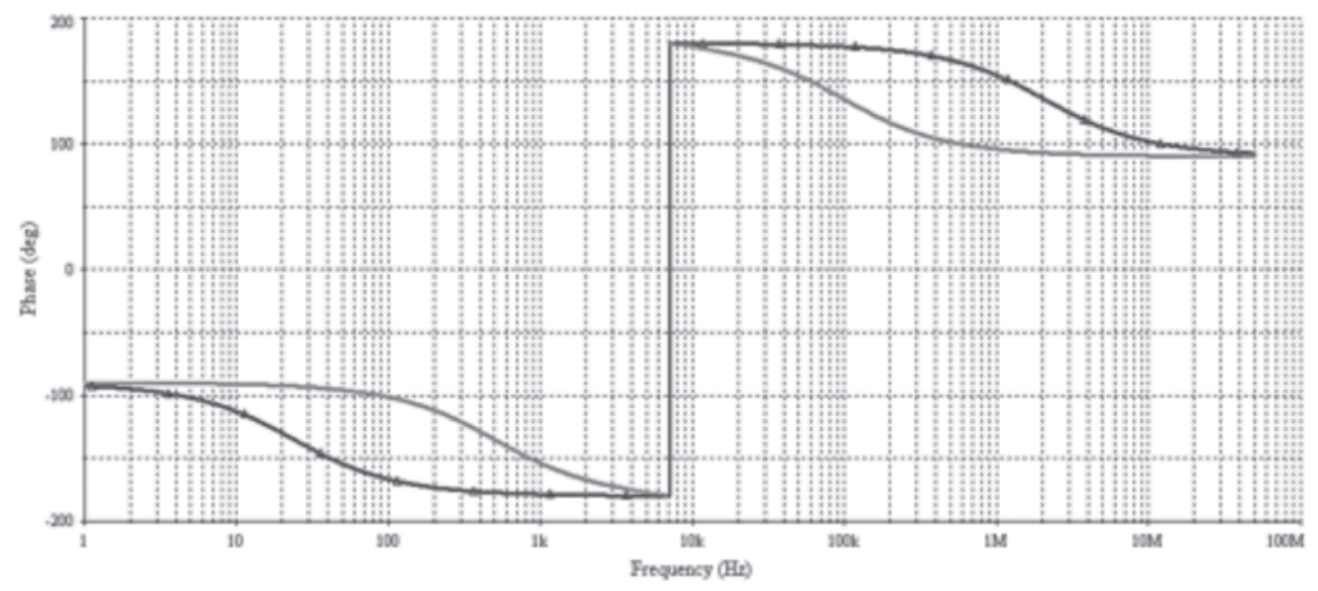

Fuente: Tomado del simulador Multisim.

\section{CONCLUSIONES}

- En la introducción de este artículo quedo planteada la siguiente pregunta, ¿Es posible obtener un tipo de realimentación negativa de lazo simple que ofrezca todas las ventajas del concepto clásico sin penalizar la ganancia? La respuesta se detalla a continuación. Durante este trabajo, y siguiendo la lógica física utilizada por Hawksford, su propuesta de un esquema de realimentación con la intención perseguida en la pregunta anterior, se llegó a un esquema que fue llamado Realimentación Negativa AS (RNAS). La RNAS fue sometida inicialmente a una serie de análisis teóricos análogos a los que comúnmente se le aplican a la realimentación negativa clásica, esto es, análisis de sensibilidad a la ganancia de lazo abierto, análisis de la respuesta en frecuencia, análisis de distorsión y análisis de desempeño frente al ruido. En el primero de los mencionados análisis, la RNAS presento un comportamiento cercano al de la realimentación negativa clásica. En el segundo análisis, la RNAS mostro un incremento en el ancho de banda de igual proporción al experimentado con la realimentación convencional. En el tercero de los análisis mencionados, la RNAS no logró reducir la distorsión producto de la no linealidad del amplificador bajo estudio. Esto se debió a que la RNAS no redujo la ganancia de rango medio del amplificador y por ende la distorsión, que está asociada a la ganancia nominal del amplificador, tampoco fue reducida, pero esto es lógicamente aceptable desde el punto de vista del funcionamiento físico del amplificador. En cuanto al cuarto de los análisis, el de desempeño frente al ruido, se demostró teóricamente que la RNAS tenía un comportamiento en este sentido fuertemente asociado a los valores de $G, A_{1}$ y $A_{2}$. El resultado obtenido no permitió llegar a una conclusión clara sobre las posibles mejoras en el desempeño de los amplificadores frente al ruido de la RNAS. Sin embargo, se restó importancia a este hecho, ya que tanto para la realimentación negativa clásica como para la RNAS, la mejora frente el ruido solo se dará si se le antepone al amplificador ruidoso uno libre de ruido, lo que constituye una situación irreal. Finalmente, queda pendiente un análisis de estabilidad para establecer cuál es la condición en la que el uso de la RNAS no hace inestables los circuitos que la implementan. Por todo lo anterior, se llega a la conclusión de que la pregunta presentada en el inicio se respondió afirmativamente en parte. Y esto es así porque en líneas generales, si fue posible llegar a un esquema que permitiera tener algunas de las ventajas de la realimentación negativa convencional, pero al costo de no lograr mejorar la distorsión en el sistema. Este resultado es lógico debido a la fuerte relación que existe entre la ganancia y la distorsión, y como el objetivo era no perder ganancia de rango medio no fue posible reducir la distorsión en el sistema. Posteriormente, los resultados teóricos fueron contrastados con simulaciones en el software de aplicación Multisim ${ }^{\circledR}$ de National Instruments y corroboraron los análisis teóricos realizados.

- Como se hizo evidente durante el artículo, las ideas aquí presentadas son apenas nacientes, por tanto no 
se intentó ser exhaustivo en cuanto al desarrollo de las mismas, sino presentar las bases del concepto central. Lo anterior deja claro que es mucho el trabajo hacia el futuro para conseguir la maduración de la idea. Se plantean entonces los siguientes trabajos potenciales: desarrollar un análisis de estabilidad riguroso, llevar a cabo las simulaciones usando técnicas de diseño de experimentos, analizar la respuesta en frecuencia de este esquema de realimentación sin asumir que el amplificador $G(s)$ tiene respuesta en frecuencia constante, e investigar las potenciales aplicaciones prácticas concretas que pueda tener la aproximación aquí presentada.

\section{REFERENCIAS}

[1] Breed, G., Feedback Fundamentals: Basic Concepts and Circuit Topologies, High Frequency Design - High Frequency Electronics, July 2006.

[2] Millman, J., Halkias, C., Integrated electronics: analog and digital circuits and systems, McGraw-Hill, p. 911, 1972.

[3] n Wiley and Son, $3^{\text {th }}$ Ed, 1996'.

[4] Karki, J. L., Designing for low distortion with highspeed op amps, Analog and Mixed-Signal Products, Analog Applications Journal, July 2001.

[5] Van Hartingsveldt, K., et. al., Influence of frequency compensation on the linearity of negative feedback amplifiers, IEEE International Symposium on Circuits and Systems ISCAS 2005, Vol. 2, 1610 - 1613, 2005.

[6] Villanueva, R.G., Aguilar, H.J., Amplifier linearisation through the use of special negative linear feedback, IEEE Proceedings on Circuits Devices and Systems, Vol. 143(1), $61-67,1996$.

[7] Krishnapura, N., Introducing negative feedback with an integrator as the central element, IEEE International Symposium on Circuits and Systems (ISCAS), 2449 - 2452, 2012.

[8] Lynch, W. A., The Stability Problem in Feedback Amplifers, Proceedings of the I.R.E., 1000-1008, September 1951.

[9] Blake, R., Sistemas electrónicos de comunicaciones, Ediciones Paraninfo S.A., $2^{\text {th }}$ Ed, 2005.

[10] Tomasí, W., Sistemas de comunicaciones electrónicas, Pearson Educación, $4^{\text {th }}$ Ed, p. 948, 2003.
[11] Xiu-Ling, W., Wen-Lan, W., Xiao-Dong, Z., Jia-Ying, Z., Study of negative feedback amplifier circuit based on Multisim, 2nd International Conference on Computer Engineering and Technology (ICCET), Vol. 6, 85-87, 2010.

[12] Jun, W., Noise analysis and design of high-frequency negative feedback amplifier using a modularized method, IEEE Asia-Pacific Conference on Circuits and Systems, IEEE APCCAS, 803 - 805, 2000.

[13] Serdijn, W.A., Setti, G., A UWB CMOS 0.13 $\mu$ m lownoise amplifier with dual loop negative feedback, IEEE International Symposium on Circuits and Systems ISCAS, $672-675,2008$.

[14] Franco, S., Design with operational amplifiers and analog integrated circuits, MacGraw-Hill, $3^{\text {th }}$ Ed, p. 680, 2002.

[15] Edinger, J. R ., High-Quality Audio Amplifier With Automatic Bias Control, Audio Engineering, June, 1947.

[16] Terman, F. E., Radio Engineering, McGraw Hill Book Company Inc, 1937.

[17] Lapatine, S., Electrónica en Sistemas de Comunicación, Editorial Limusa S.A., p. 200, 1996.

[18] Terman, F. et. al., Applications of Negative Feedback, Proceedings of the I.R.E, October 1939.

[19] Garzón-Gaitán, A. J., Introducción a la electrónica de las radiofrecuencias, Escuela Colombiana de Ingeniería, $1^{\text {st }}$ Ed, Octubre 2008.

[20]Galocha, B., Fernández, J., et. al., Electrónica de comunicaciones, Pearson - Prentice Hall, 2010.

[21] Xiaoliang, C., Jianli, X., A compact output stage for low-power low-distortion amplifier with diode negative feedback, International Conference on Anti-Counterfeiting Security and Identification in Communication (ASID), 97- 100, 2010.

[22] Sedra, A., Smith, K.C., Microelectronics Circuits, Oxford University Press, $4^{\text {th }}$ Ed, 1996.

[23]Malvino, A., Bates, D., Principios de Electrónica, McGraw-Hill, $7^{\text {th }}$ Ed. 2007.

[24] Bode, H. W., Relations between Attenuation and Phase in Feedback Amplifier Design, Bell System Tech. J., vol. 19, July 1940.

[25] Blackman, R. B., Effect of Feedback on Impedance, Bell System Tech. J., vol. 23, Octuber 1943. 
[26] Black, H. S., Stabilized Feedback Amplifiers, Bell System Tech. J., January 1934.

[27] Bode, H. W., Network Analysis and Feedback Amplifier Design, D. Van Nostrand Company, Princeton, N.J., September 1945.

[28] Blecher, H., Design Principles for Single Loop Transistor Feedback Amplifiers, IRE Trans. Circuit Theory, vol. CT-4 N 5, September 1957.

29] Marshall-Leach, W., Feedback Amplifiers, Georgia Institute of Technology, School of Electrical and Computer Engineering, 2009.

[30] Rashid, M., Circuitos microelectrónicos: análisis y diseño, Ediciones Paraninfo S.A., 2000.

[31] Nikolic, B., Slavoljub, M., A general method of feedback amplifier analysis, Proceedings of the IEEE International Symposium on Circuits and Systems ISCAS, Vol. 3, $415-418,1998$.

[32] Van der Horst, M.J., Linnenbank, A.C., Van Staveren, A., Amplitude-modulation detection in single-stage negative-feedback amplifiers due to interfering out-of-band signals, IEEE Transactions on Electromagnetic Compatibility, Vol. 47(1), 34- 44, 2005.

[33] Totev, E.D., Verhoeven, C.J.M., Out of band interference measurement of negative feedback amplifiers, 6th International Conference on ASIC, ASICON. Vol. 2, 676- 680, 2005.

[34] Hawksford, M. J., Distortion Correction in Audio Power Amplifiers, JAES, vol. 29, No. 1/2., 27-30, Jan/Feb 1981.
[35] Hawksford, M. J., Reduction of Transistor Slope Impedance Dependent Distortion in Large-Signal Amplifiers, JAES, vol. 36, No. 4, 213-222, April 1988.

[36] Hawksford, M. J., Distortion Correction Circuits for Audio Amplifiers, JAES, vol. 29, No. 7/8, 503-510, July/August 1981.

[37] Ghausi, M. S., Electronic Devices and Circuits: Discrete and Integrated, Holt, Rinehart \& Winston, New York, 1985.

[38] Schilling, D., Belove, H., Electronic Circuits Discrete and 'Integrated, McGraw - Hill Book Company, New York, 1979.

[39] Soclof, S., Analog, Integrated Circuits, Prentice-Hall, Englewood Cliffs, N.J, 1985.

[40] Chenghua, W., Hua, B., Research on Nonlinear Distortion in Amplifiers, Transactions of Nanjing University of Aeronautics \& Astronautics, Vol. 17(2), December 2000.

[41] Gray, P., Meyer, R., MOS operational amplifier design: A tutorial overview, IEEE Journal of Solid-State Circuits, Vol. SC-17(6), 969-982, December 1982.

[42] Solomon, J., The monolithic op amp: A tutorial study, IEEE Journal of Solid-State Circuits, Vol. SC-9(6), 314 332, December 1974.

[43] Self, D., Audio Power Amplifier Design Handbook, February 2011.

[44] Jardón, H., Golovin, O., Receptores para Sistemas de Radiocomunicación, Alfaomega, 1998. 